\title{
What Characterizes Fluid Intake Patterns across the World?
}

\author{
Clémentine Morin $^{a}$ Joan Gandy ${ }^{b}$ Luis A. Moreno ${ }^{c, d, e ~ S t a v r o s ~ A . ~ K a v o u r a s ~}{ }^{f}$ \\ Homero Martinez $^{g}$ Jordi Salas-Salvado ${ }^{e, h, i}$ Jeanne Bottin ${ }^{a}$ \\ aDanone Research, Palaiseau, France; breelance Dietitian, London, UK; ${ }^{c}$ Growth, Exercise, Nutrition and \\ Development (GENUD Research Group, Instituto Agroalimentario de Aragón (IA2), Universidad de Zaragoza, \\ Zaragoza, Spain; dInstituto de Investigación Sanitaria Aragón (IIS Aragón), Zaragoza, Spain; 'Consorcio CIBER, M.P. \\ Fisiopatología de la Obesidad y Nutrición (CIBERObn), Instituto de Salud Carlos III (ISCIII), Madrid, Spain; ' Hydration \\ Science Lab, College of Health Solutions, Arizona State University, Phoenix, AZ, USA; ${ }^{\circ H o s p i t a l ~ I n f a n t i l ~ d e ~ M e ́ x i c o ~}$ \\ Federico Gómez, México City, Mexico; ${ }^{\text {h}}$ Departament de Bioquímica i Biotecnologia, Universitat Rovira i Virgili, \\ Unitat de Nutrició Humana, Reus, Spain; 'Institut d'Investigació Sanitària Pere Virgili (IISPV), Hospital Universitari San \\ Joan de Reus, Reus, Spain
}

\section{Keywords}

Cluster analysis $\cdot$ Drinking patterns $\cdot$ Children $\cdot$ Beverages intake $\cdot$ Hydration

\begin{abstract}
Introduction: Total fluid intake and the type of fluids consumed have been reported by many studies [1-3] and have shown that while an individual may be drinking sufficiently, in terms of volume, to meet or exceed recommendations on fluid intake, there may be a wide variety of combinations of fluids within that total volume [4-6]. Moreover, considering only volume and fluid types may limit the interpretation of the data [7]. In a novel approach, we propose to analyze and understand fluid intake patterns as opposed to only fluid volume or types. The primary aim of this study was to identify patterns of fluid intake in children and adolescents from 6 countries: Argentina, Brazil, Mexico, Uruguay, China, and Indonesia. The secondary aim was to characterize those fluid intake patterns. Methods: A validated 7-day fluid specific record (Liq.In7 record) [8] was used to collect primary data on fluid intake amongst children and adolescents (10-17 years;
\end{abstract}

$N=1,781)$. To identify relatively distinct clusters of subjects based on 8 fluid types (water, milk and its derivatives, hot beverages, sugar-sweetened beverages [SSB], 100\% fruit juices, artificial/nonnutritive sweetened beverages, alcoholic beverages, and other beverages), a cluster analysis (partitioning around k-medoids algorithm) was used. Clusters were then characterized according to their socio-demographic and lifestyle indicators. Results: The 6 clusters identified (Fig. 1) were low drinkers - SSB $(n=523)$, low drinkers - water and milk ( $n=615)$, medium mixed drinkers $(n=914)$, high drinkers - SSB $(n=513)$, high drinkers - water $(n=352)$, and very high drinkers - water $(n=264)$. Country of residence was the dominant characteristic, followed by socioeconomic level, in all 6 patterns. Conclusion: Fluid intake patterns among children are primarily driven by water and SSB. In addition to country, socio-demographic and lifestyle factors determined the characteristics of each cluster. Therefore, interventions aiming to encourage healthier fluid intake behavior need to target and be tailored to a particular subpopulation.

(C) 2022 The Author(s)

Published by S. Karger AG, Basel karger@karger.com www.karger.com/anm

Karger $\stackrel{\text { ' }}{=}$

BOPEN ACCESS
(C) 2022 The Author(s)

Published by S. Karger AG, Basel

This article is licensed under the Creative Commons Attribution 4.0 International License (CC BY) (http://www.karger.com/Services/ OpenAccessLicense). Usage, derivative works and distribution are permitted provided that proper credit is given to the author and the original publisher.
Correspondence to:

Clémentine Morin, clementine.morin@danone.com 


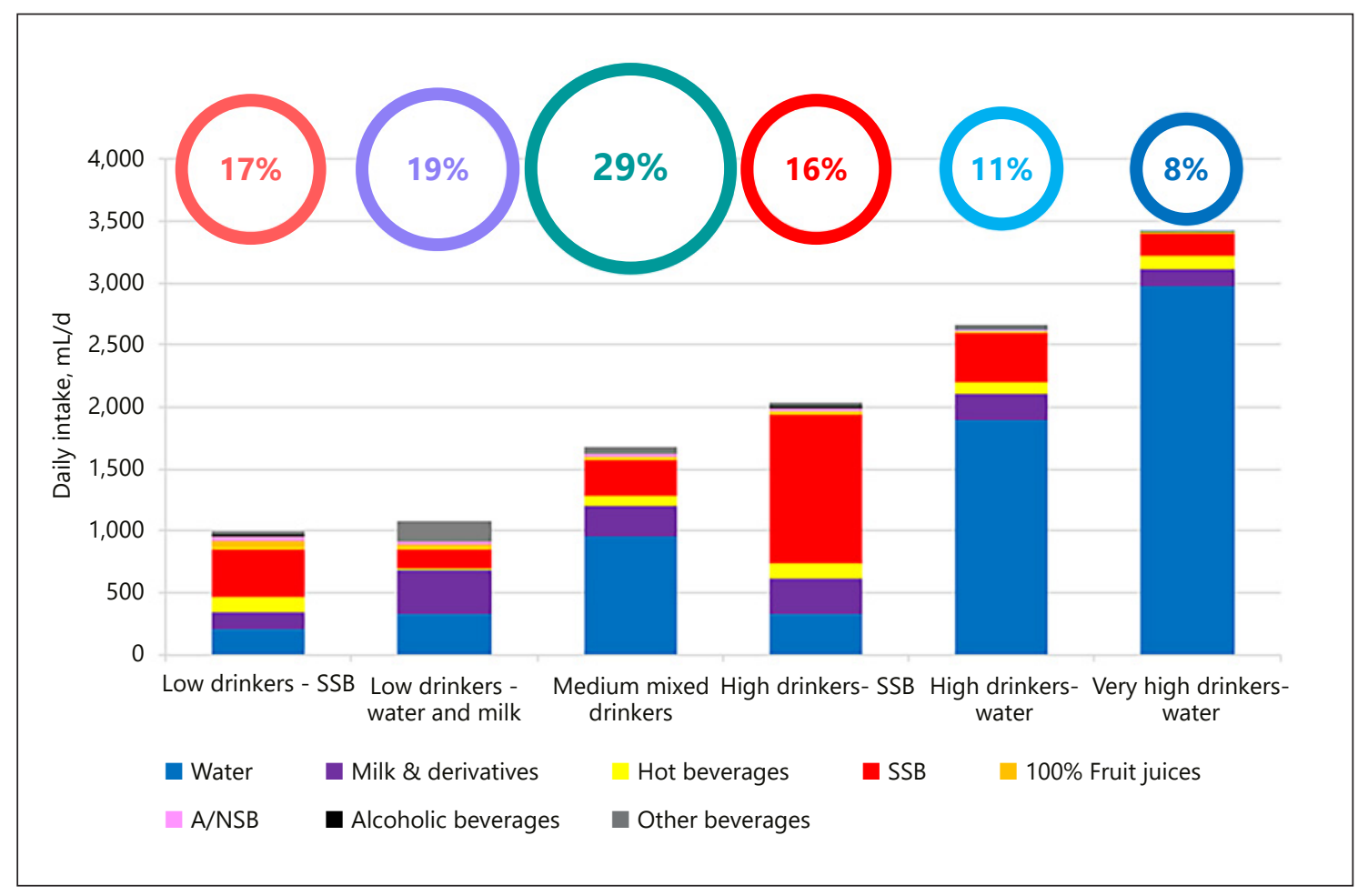

Fig. 1. Mean daily intake of different fluid types (mL/day) of each cluster among children and adolescents. SSB, sugar-sweetened beverages.

\section{Acknowledgment}

The authors wish to thank the participants who took part in the original data collection and to acknowledge the role of IPSOS in participant recruitment and data collection. They also thank Isabelle Guelinckx and Rémi Brazeilles for their support in the conduct of this analysis and the critical review of the manuscript.

\section{Statement of Ethics}

Study approval statement: The protocol of the surveys was reviewed and approved by the Institutional Review Board, Office of Research Compliance of the University of Arkansas (IRB Protocol \# 14-12-376). Consent to participate statement: All the participants gave their written informed consent prior the inclusion in the study. All data were recorded and analyzed anonymously.

\section{Conflict of Interest Statement}

C.M. and J.B. are full-time employees of Danone Research. J.G. is a member of the Scientific Committee of Hydration for Health and received consultancy fees from Danone Research. J.S.-S. partially supported by ICREA under the ICREA Academia programme. S.A.K., L.M., and H.M. have received research grants from Danone Research.

\section{Funding Sources}

The study received no funding.

\section{Data Availability Statement}

The datasets used for the purpose of this analysis are available from the corresponding author upon reasonable request. 


\section{References}

1 Vieux F, Maillot M, Constant F, Drewnowski A. Water and beverage consumption patterns among 4 to 13-year-old children in the United Kingdom. BMC Public Health. 2017;17:479.

2 Iglesia I, Guelinckx I, De Miguel-Etayo PM, González-Gil EM, Salas-Salvadó J, Kavouras SA, et al. Total fluid intake of children and adolescents: cross-sectional surveys in 13 countries worldwide. Eur J Nutr. 2015; 54(Suppl 2):57-67.

3 Popkin BM, Barclay DV, Nielsen SJ. Water and food consumption patterns of US adults from 1999 to 2001. Obes Res. 2005; 13:214652.
4 Nissensohn M, Sánchez-Villegas A, Ortega RM, Aranceta-Bartrina J, Gil Á, GonzálezGross $\mathrm{M}$, et al. Beverage consumption habits and association with total water and energy intakes in the Spanish population: findings of the ANIBES Study. Nutrients. 2016;8:232

5 Guelinckx I, Iglesia I, Bottin JH, De MiguelEtayo P, González-Gil EM, Salas-Salvadó J, et al. Intake of water and beverages of children and adolescents in 13 countries. Eur J Nutr. 2015;54(Suppl 2):69-79.
6 Guelinckx I, Ferreira-Pêgo C, Moreno LA, Kavouras SA, Gandy J, Martinez H, et al. Intake of water and different beverages in adults across 13 countries. Eur J Nutr. 2015;54(Suppl 2):45-55.

7 Miller JM, Guo Y, Rodseth SB. Cluster analysis of intake, output, and voiding habits collected from diary data. Nurs Res. 2011;60: 115-23.

8 Johnson EC, Péronnet F, Jansen LT, CapitanJiménez C, Adams JD, Guelinckx I, et al. Validation testing demonstrates efficacy of a 7-day fluid record to estimate daily water intake in adult men and women when compared with total body water turnover measurement. J Nutr. 2017;147:2001-7. 